OESOPHAGUS

\title{
Preoperative chemoradiotherapy for oesophageal cancer: a systematic review and meta-analysis
}

\author{
F Fiorica, D Di Bona, F Schepis, A Licata, L Shahied, A Venturi, A M Falchi, A Craxì, C Cammà
}

Gut 2004;53:925-930. doi: 10.1136/gut.2003.025080

Background: The benefit of neoadjuvant chemoradiotherapy in oesophageal cancer has been extensively studied but data on survival are still equivocal.

Objective: To assess the effectiveness of chemoradiotherapy followed by surgery in the reduction of mortality in patients with resectable oesophageal cancer.

Methods: Computerised bibliographic searches of MEDLINE and CANCERLIT (1970-2002) were supplemented with hand searches of reference lists.

Study selection: Studies were included if they were randomised controlled trials (RCTs) comparing preoperative chemoradiotherapy plus surgery with surgery alone, and if they included patients with resectable histologically proven oesophageal cancer without metastatic disease. Six eligible RCTs were identified and included in the meta-analysis.

Data extraction: Data on study populations, interventions, and outcomes were extracted from each RCT according to the intention to treat method by three independent observers and combined using the DerSimonian and Laird method.

See end of article for authors' affiliations

Results: Chemoradiotherapy plus surgery compared with surgery alone significantly reduced the three year mortality rate (odds ratio (OR) 0.53 (95\% confidence interval (CI) $0.31-0.93$ ); $p=0.03$ ) (number needed to treat $=10$ ). Pathological examination showed that preoperative chemoradiotherapy downstaged the tumour (that is, less advanced stage at pathological examination at the time of surgery)

Correspondence to: Dr Calogero Cammà, Via Alcide De Gasperi 50, 90100 Palermo, Italy; camma@ibim.cnr.it

Accepted for publication 9 January 2004 compared with surgery alone (OR $0.43(95 \% \mathrm{Cl} 0.26-0.72) ; p=0.001)$. The risk for postoperative mortality was higher in the chemoradiotherapy plus surgery group (OR $2.10(95 \% \mathrm{Cl} 1.18-3.73)$; $\mathrm{p}=0.01$ ).

Conclusions: In patients with resectable oesophageal cancer, chemoradiotherapy plus surgery significantly reduces three year mortality compared with surgery alone. However, postoperative mortality was significantly increased by neoadjuvant chemoradiotherapy. Further large scale multicentre RCTs may prove useful to substantiate the benefit on overall survival.
W orldwide, oesophageal cancer ranks fifth in mortality rate among tumour sites. ${ }^{1}$ The European weighted survival, calculated from the pool of all cancer registries, was $33 \%$ at one year and $10 \%$ at five years. ${ }^{2}$ The three year survival rate of patients with local-regional oesophageal cancer who have undergone curative resection remains low (approximately $20 \%$ ) with a high postoperative mortality rate ranging from $3 \%$ to $10 \% .^{3-6}$ Therefore, oesophageal cancer is a treatable but rarely curable disease. Improved and standardised surgical techniques as well as advances in supportive care have contributed to an increase in the rate of curative resection. ${ }^{5}$ Nevertheless, a plateau in the effectiveness of surgical resection may well have been reached and further improvement in survival from a single modality approach seems unlikely. ${ }^{6}$

Recently, neoadjuvant therapy has become the focus of interest in an effort to prolong survival and reduce recurrence rates in patients with oesophageal cancer. Studies have indicated that preoperative radiotherapy increases the rate of resectability but a meta-analysis of these randomised controlled trials (RCTs) failed to show a statistically significant benefit on survival. ${ }^{7}$ Preoperative chemotherapy trials have shown conflicting results even in the most recent large scale RCTs. ${ }^{8-11}$ The potential activity of chemotherapy against micrometastases as well as the radiosensitising properties of some chemotherapeutic agents has led to the treatment of patients with a combination of radiotherapy and chemotherapy. Since the first RCT of preoperative chemoradiotherapy (CRT) appeared in 1992, ${ }^{12}$ several RCTs have been published. ${ }^{13-22}$ The results of these trials are inconclusive or conflicting because of the relatively small samples. Additionally, it is difficult to draw general conclusions from them because of differences in patient characteristics and treatment regimens. A recently published RCT performed at the University of Michigan ${ }^{17}$ concluded that preoperative CRT did not improve survival compared with surgery alone. The aim of this systematic review and meta-analysis is to determine if there is a benefit of preoperative CRT compared with surgery alone.

\section{METHODS}

\section{Selection of randomised trials}

The primary source of the reviewed studies was MEDLINE and CANCERLIT, including non-English sources, with the following medical subject headings: oesophageal cancer, chemoradiotherapy, radiotherapy, chemotherapy, RCT, randomised, and clinical trial. The search included literature published through to December 2002. The computer search was supplemented with manual searches of reference lists for all available review articles, primary studies, abstracts from meetings, and bibliographies of books. We have contacted the investigators of an Australian trial, which was reported twice

Abbreviations: CRT, chemoradiotherapy; RCTs, randomised controlled trials; OR, odds ratio; NNT, number of patients needed to treat; $\mathrm{NNH}$, number of patients needed to harm; BED, biologically effective dose; $\mathrm{CT}$, computed tomography 
as an abstract, ${ }^{19}{ }^{20}$ in order to obtain data on randomisation and survival.

Studies were included in the meta-analysis if they were RCTs comparing preoperative CRT plus surgery with surgery alone, if they included patients with resectable histologically proven squamous cell carcinoma or adenocarcinoma of the oesophagus without metastatic disease, and if mortality was assessed as an outcome measure of the effect of the treatment. Among the 11 studies reviewed, six RCTs met the inclusion criteria. ${ }^{12-17}$ Five studies ${ }^{18-22}$ were excluded because they were published as a preliminary report or abstract ${ }^{182122}$ and subsequently published as a final paper, or because they were published as an abstract, not reporting survival data. ${ }^{19}{ }^{20}$ As all the trials reported as abstracts ${ }^{2122}$ were subsequently published as full papers, this meta-analysis included only peer reviewed reports.

This meta-analysis was performed according to the QUOROM statement. ${ }^{23}$

\section{Review of the trials}

The trials were first reviewed using a list of predefined pertinent issues that concerned the characteristics of patients and treatments. To assess the methodological quality of RCTs, the two domains of blinding and handling withdrawals and dropouts, using the definitions given by Nicolucci and colleagues, ${ }^{24}$ were used, as suggested by Jüni and colleagues..$^{25}$ Each RCT was evaluated and classified by three independent investigators (FF, AV, DDB). Discrepancies among reviewers were infrequent (overall interobserver variations $<10 \%)$, and were resolved by discussion.

\section{Statistical methods}

The crude rate of three year overall mortality was assessed as a measure of treatment effect. These data were available in three RCTs ${ }^{15-17}$ In the remaining three trials ${ }^{12-14}$ we used the KaplanMeier estimates of the three year mortality in the treated and control groups reported in the text. Moreover, to assess the downstaging effect of CRT (that is, the probability of having a less advanced stage of the disease at pathological examination at the time of surgery), we separated patients with negative nodes and patients with positive nodes on pathological examination at the time of surgery. As a measure of treatment benefit, we compared the proportion of patients observed in the treated and control groups who were classified as stage 0 , 1 , or $2 \mathrm{a}$ according to the American Joint Committee on Cancer. ${ }^{26}$ Downstaging by chemoradiotherapy was also used as a measure of treatment effect on pathological response at the time of surgery. Furthermore, we analysed the 90 day inhospital mortality (postoperative mortality).

Evaluation of therapeutic effectiveness was performed by an intention to treat method. When not reported in the trial, response rate, according to the intention to treat method, was calculated. The number of patients who discontinued their original irradiation regimen because of side effects was also recorded. To combine results from individual trials, we used the proportion of events observed in the treated and control groups. With these observed proportions of events, the odds ratio (OR) was computed for each trial.

The overall OR among the frequencies of the events in both the chemoradiotherapy plus surgery group and the surgery alone group was calculated with models based on both fixed effects and random effects assumptions. In addition to within study variance, the random effects model considers heterogeneity among studies. Because of the different clinical settings and groups of subjects analysed and because the tests for heterogeneity lack statistical power due to the few studies included in this meta-analysis, we have presented the results of random effects models, according to DerSimonian and Laird. ${ }^{27}$ The $95 \%$ confidence interval (95\% CI) of the odds ratio was also calculated. The overall OR was tested for significance using a Mantel-Haenszel $\chi^{2}$ test. ${ }^{28}$

Moreover, we in turn excluded each study to ensure that no single study would be solely responsible for the significance of any result (so-called robust analysis). All our analyses were computed using Metaview 4.0. The number of patients needed to treat (NNT) to prevent one death, and the number of patients needed to harm $(\mathrm{NNH})$ to prevent one death by postoperative mortality, which both derive from the inverse of the risk difference, were also used as a measure of treatment benefit and safety. ${ }^{29}$

To improve the comparability of the different therapeutic regimens and to assess the relationship between radiation dose and survival benefit, the biologically effective dose (BED), corrected for time of the various radiation schedules, was estimated..$^{30}$ In the Scandinavian trial, ${ }^{12}$ three different treatment arms were compared with the same surgery arm as the control. We included in the analysis only the chemoradiation treatment arm of this RCT, using effect size estimates calculated from observations on that measure. Therefore, the statistical analysis used only independent estimators of effect size. ${ }^{31}$

Subgroup analyses were used to explore and explain the diversity among results of different studies. We used two stratifying variables: adenocarcinoma versus squamous cell carcinoma and BED $>35$ versus $\mathrm{BED} \leqslant 35$. A $\chi^{2}$ test for interaction $^{32}$ was used to examine whether the effect of treatment varied significantly between subgroups.

\section{Source of support}

This meta-analysis was entirely supported by the authors' respective institutions.

Table 1 Patients characteristics of trials included in the meta-analysis

\begin{tabular}{|c|c|c|c|c|c|c|c|c|c|}
\hline & & \multirow{2}{*}{$\begin{array}{l}\text { Accrual } \\
\text { period (y) }\end{array}$} & \multirow{2}{*}{\multicolumn{2}{|c|}{$\begin{array}{l}\text { Patients randomised } \\
\text { (n) }\end{array}$}} & \multirow{2}{*}{$\begin{array}{l}\text { Male } \\
(\%)\end{array}$} & \multirow{2}{*}{$\begin{array}{l}\text { Mean age } \\
\text { (y) }\end{array}$} & \multicolumn{2}{|c|}{ Histology (\%) } & \multirow[b]{2}{*}{ Preoperative stage (\% of patients) } \\
\hline \multicolumn{2}{|c|}{ Study (reference)* } & & & & & & SCC & ADENO & \\
\hline \multirow[t]{2}{*}{1} & Nygaard et $a l^{22}$ & $1983-88$ & $\mathrm{CRT}+\mathrm{S}$ & 53 & 70 & 60.1 & 100 & 0 & $1-11(100)$ \\
\hline & & & S & 50 & 75 & 61.4 & 100 & 0 & $1-I \mid(100)$ \\
\hline \multirow[t]{2}{*}{2} & Le Prise et $a l^{p^{3}}$ & 1988-91 & $\mathrm{CRT}+\mathrm{S}$ & 41 & 93 & 56 & 100 & 0 & I (12.2); II (65.8); not specified (22) \\
\hline & & & S & 45 & 93 & 59 & 100 & 0 & I (35.5); II (55.6); not specified (8.9) \\
\hline \multirow[t]{2}{*}{3} & Apinop et $a l^{14}$ & 1986-92 & $\mathrm{CRT}+\mathrm{S}$ & 35 & 80 & 59.6 & 100 & 0 & Ilb (17.1); III (82.9) \\
\hline & & & $\mathrm{S}$ & 34 & 76 & 59.8 & 100 & 0 & Ilb (14.8); III (82.3); not specified (5.9) \\
\hline \multirow[t]{2}{*}{4} & Walsh et $a l^{15}$ & 1990-95 & $\mathrm{CRT}+\mathrm{S}$ & 58 & 67 & 65 & 0 & 100 & NR \\
\hline & & & $\mathrm{S}$ & 55 & 80 & 65 & 0 & 100 & NR \\
\hline \multirow[t]{2}{*}{5} & Bosset et $a l^{16}$ & 1989-95 & $\mathrm{CRT}+\mathrm{S}$ & 151 & 90 & 57 & 100 & 0 & I (15.9); Ila (56.9); Ilb (21.8) not specified (8) \\
\hline & & & S & 146 & 96 & 57 & 100 & 0 & I (17.1); Ila (56.2); Ilb (21.9) not specified (4.8) \\
\hline \multirow[t]{2}{*}{6} & Urba et al ${ }^{77}$ & 1989-94 & $\mathrm{CRT}+\mathrm{S}$ & 50 & 84 & 62 & 26 & 74 & I-Ila (60); IIb-III (40) \\
\hline & & & S & 50 & 86 & 64 & 24 & 76 & I-Ila (56); IIb-III (44) \\
\hline
\end{tabular}

*For expansion of the study names, see corresponding reference.

CRT+S, chemoradiotherapy plus surgery; S, surgery; SCC, squamous cell carcinoma; ADENO, adenocarcinoma; NR, not reported. 
Table 2 Therapeutic regimen of all trials included in the meta-analysis

\begin{tabular}{|c|c|c|c|c|c|c|c|c|c|c|}
\hline & & \multicolumn{5}{|c|}{ Radiotherapy } & \multicolumn{2}{|c|}{ Chemotherapy } & \multicolumn{2}{|l|}{ Surgery } \\
\hline Study (reference) & (reference) $\ddagger$ & $\begin{array}{l}\text { Total dose } \\
\text { (Gy) }\end{array}$ & $\begin{array}{l}\text { Fractions } \\
\text { (No/days) }\end{array}$ & $\begin{array}{l}\text { Daily dose } \\
\text { (Gy) }\end{array}$ & $\begin{array}{l}\text { BED } \\
\text { (Gy) }\end{array}$ & $\begin{array}{l}\text { BED corrected } \\
\text { by time (Gy) }\end{array}$ & Drugs & $\begin{array}{l}\text { Dosage } \\
\left(\mathrm{mg} / \mathrm{m}^{2}\right)\end{array}$ & $\begin{array}{l}\text { Schedules } \\
\text { (day) }\end{array}$ & $\begin{array}{l}\text { Interval between } \\
\text { end of irradiation } \\
\text { and surgery (weeks) }\end{array}$ \\
\hline 1 & Nygaard et $a l^{2}$ & 35 & $20 / 28$ & 1.75 & 41.12 & $26.7^{*}$ & $\begin{array}{l}\text { CDDP } \\
\text { BLM }\end{array}$ & $\begin{array}{l}20 \\
5\end{array}$ & $1-5 ; 15-19$ & 3 \\
\hline 2 & Le Prise et $a l^{13}$ & 20 & $10 / 12$ & 2 & 24 & $17.8^{*}$ & $\begin{array}{l}\text { CDDP } \\
5 F U\end{array}$ & $\begin{array}{l}100 \\
600\end{array}$ & $\begin{array}{l}1 ; 21 \\
2-5 ; 22-25\end{array}$ & 2.5 \\
\hline 3 & Apinop et al ${ }^{1 / 4}$ & 40 & $20 / 28$ & 2 & 48 & $33.6^{*}$ & $\begin{array}{l}\text { CDDP } \\
\text { 5FU }\end{array}$ & $\begin{array}{l}100 \\
1000\end{array}$ & $\begin{array}{l}1 ; 29 \\
1-4 ; 29-32\end{array}$ & 4 \\
\hline 4 & Walsh et al ${ }^{15}$ & 40 & $15 / 21$ & 2.67 & 50.7 & $42.6+$ & $\begin{array}{l}\text { CDDP } \\
5 F U\end{array}$ & $\begin{array}{l}75 \\
15 \mathrm{mg} / \mathrm{kg}\end{array}$ & $\begin{array}{l}7 ; 42 \\
1-5 ; 36-40\end{array}$ & 2 \\
\hline 5 & Bosset et al ${ }^{16}$ & 37 & $10 / 24$ & 3.7 & 50.7 & $38.4^{*}$ & CDDP & 80 & $0-2 ; 19-21$ & $2-4$ \\
\hline \multirow[t]{3}{*}{6} & Urba et al ${ }^{17}$ & 45 & $30 / 21$ & $1.5 \times 2$ & 51.7 & $\begin{array}{l}43.6 \dagger \\
\text { adenoca. }\end{array}$ & CDDP & 20 & $1-5 ; 17-21$ & 3 \\
\hline & & & & & & $40.9^{*}$ & $5 F U$ & 300 & $1-21$ & \\
\hline & & & & & & & VNB & 1 & $1-4 ; 17-20$ & \\
\hline
\end{tabular}

${ }^{*}$ Tpot $_{\text {squamo }}=4.5$ days $^{33} ;+T_{\text {Tpot }}{ }_{\text {adeno }}=6$ days, ${ }^{33} \alpha=0.3$

BED, biological equivalent dose; CDDP, cisplatin; BLM, bleomycin; 5-FU, 5-fluorouracil; VNB, vinblastin.

\#For expansion of the study names, see corresponding reference.

\section{RESULTS}

\section{Features of the RCTs}

The main features of the trials included in the meta-analysis are shown in table 1 . The six RCTs included 764 patients, 385 of whom received CRT before surgery. The percentage of males ranged from $78 \%^{14}$ to $93 \% .^{13}{ }^{16}$ The criteria for inclusion were uniform in all but one RCT, which included only patients with locally advanced but otherwise operable tumours. ${ }^{14}$ Only two studies ${ }^{12}{ }^{16}$ were multicentre trials. The sample size of each RCT varied greatly, ranging from $69^{14}$ to $297^{16}$ patients. No adequate blinding was used in two trials $^{1214}$ and the method of randomisation was not clearly reported in the remaining four studies. ${ }^{12-14}$ Three studies ${ }^{12-14}$ did not clearly define criteria for handling withdrawals.

The preoperative staging procedures were uniform in all RCTs. In four RCTs ${ }^{12} 141617$ all patients underwent computed tomography (CT) while in the study of Le Prise and colleagues, ${ }^{13}$ and in the Irish trial ${ }^{15}$ CT was performed in selected patients only. Data on preoperative staging of the tumour were similar in all RCTs, except the small study of Apinop and colleagues ${ }^{14}$ in which stage I oesophageal cancer was excluded (table 1). In all RCTs no significant differences were found in the preoperative stage of the tumour between treated and control groups.

A transthoracic resection was performed in all RCTs but one ${ }^{17}$ in which a transhiatal oesophagectomy was employed. The rate of resection (curative or palliative) reported in all
RCTs ranged from $82 \%{ }^{12}$ to $97 \% .{ }^{14}{ }^{17}$ The proportion of patients who underwent resection classified as curative (defined by the surgeon as margins of the resected tissue free of tumour) was reported in only three RCTs, ${ }^{12}{ }^{13} 17$ ranging from $46 \%$ to $97 \%$.

Pathological stage was assessed in all RCTs at the time of operation according to the American Joint Committee on Cancer. The rate of pathological stage III oesophageal cancer in patients treated by surgery alone was comparable in all RCTs, ranging from $50 \%{ }^{16}$ to $78 \% .{ }^{15}$ In four RCTs, ${ }^{12-14}{ }^{16}$ only patients with squamous cell carcinoma were included, while in the study by Walsh and colleagues ${ }^{15}$ all subjects had adenocarcinoma, and in the RCT by Urba and colleagues ${ }^{17}$ patients with either squamous cell carcinoma or adenocarcinoma were included (table 1).

The therapeutic regimens of RCTs included in the metaanalysis are shown in table 2 . Considerable heterogeneity was observed both in radiotherapy and chemotherapy protocols among the studies. A large variability in irradiation schedules between trials was found in: (a) the total dose, ranging between $20^{13}$ and $45^{17}$ Gray (Gy); (b) the daily dose, ranging between $1.75^{12}$ and $3.70^{16} \mathrm{~Gy}$; and (c) the number of fractions, ranging between $10^{16}$ and $30^{17}$ given over 24 and 21 days, respectively.

Variability in the chemotherapy protocol between trials was found in: (a) the number and type of chemotherapeutic agents administered in combination with cisplatin; and

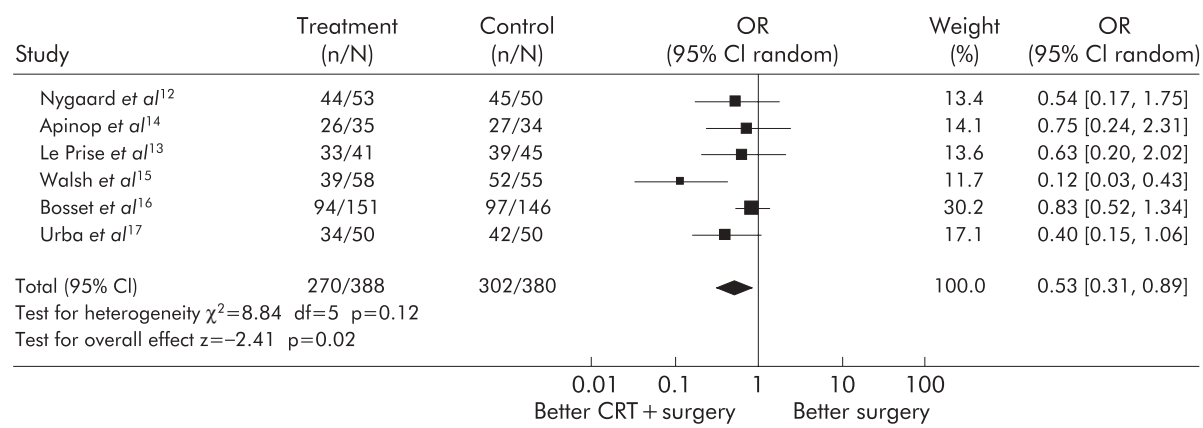

Figure 1 Meta-analysis of six randomised controlled trials of preoperative chemoradiotherapy (CRT) for resectable oesophageal carcinoma using a random effects model. The odds ratio (OR) and $95 \%$ confidence interval $(\mathrm{Cl})$ for the effect of treatment on three year overall mortality are shown on a logarithmic scale. Studies are arranged by publication year. 


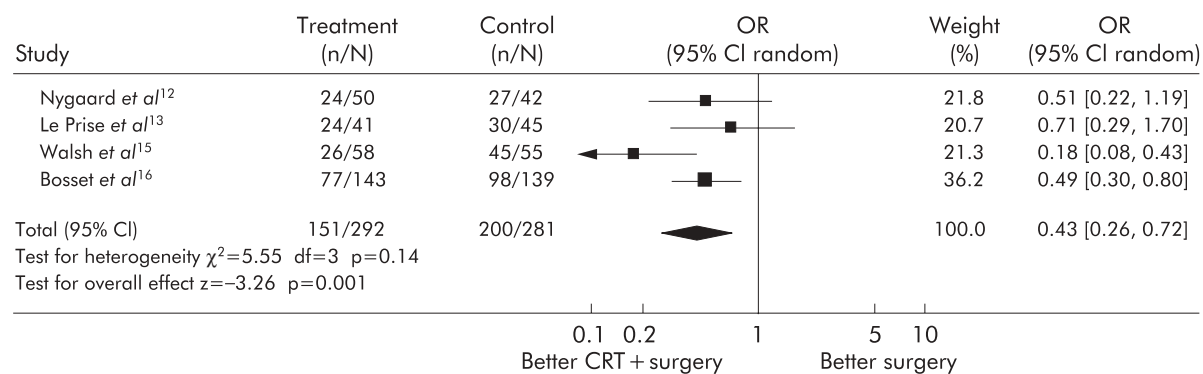

Figure 2 Meta-analysis of four randomised controlled trials of preoperative chemoradiotherapy (CRT) for resectable oesophageal carcinoma using a random effects model. The odds ratio (OR) and $95 \%$ confidence intervals $(\mathrm{Cl})$ for the effect of treatment on downstaging (defined as a lower probability to have an advanced stage of cancer at pathological examination at the time of surgery) are shown on a logarithmic scale. Studies are arranged by publication year.

(b) the dose and scheduling of chemotherapeutic drugs (table 2).

In one RCT, ${ }^{12}$ chemotherapy and radiotherapy were given sequentially with radiotherapy starting three weeks after completion of chemotherapy. In the remaining five RCTs, CRT was given simultaneously (concomitant strategy) with daily chemotherapy ${ }^{1415} 17$ or with intermittent chemotherapy. ${ }^{13} 16$

\section{Overall mortality}

The effect of adjuvant CRT on overall mortality (six RCTs: 764 patients, 573 deaths) is shown in fig 1 . Although the effect of treatment on total mortality favoured CRT in all the six trials, a statistically significant difference was observed in only one. ${ }^{15}$ However, the pooled estimate of the treatment effect was statistically significant (OR 0.53 (95\% CI $0.31-0.92)$; $\mathrm{z}=-2.23 ; \quad \mathrm{p}=0.03) \quad(\mathrm{NNT}=10)$. Similar results were obtained when a fixed effect model was used (OR 0.61 (95\% CI 0.43-0.85); $\mathrm{z}=-2.89 ; \mathrm{p}=0.004$ ).

Robust analysis showed that the five trials remaining after exclusion of either the trial by Walsh and colleagues ${ }^{15}$ or by Urba and colleagues ${ }^{17}$ lost statistical significance for overall mortality (excluding the trial by Walsh: OR 0.73 (95\% CI $0.51-1.05), \mathrm{z}=-1.68 \mathrm{p}=0.093$; excluding the trial by Urba: OR 0.55 (95\% CI 0.29-1.06), $\mathrm{z}=-1.78 ; \mathrm{p}=0.074$ ).

We performed subgroup analyses to evaluate whether there was evidence of a different effect of CRT in predefined subgroups of patients. For overall mortality, the pooled OR was statistically significant in patients with adenocarcinoma (OR 0.24 (95\% CI 0.07-0.78); $\mathrm{z}=-2.36, \mathrm{p}=0.018$ ) but not in those with squamous cell carcinoma (OR 0.81 (95\% CI $0.55-1.19) ; \mathrm{z}=-1.07, \mathrm{p}=0.29$ ), with a significant interaction $\left(\chi^{2}\right.$ for interaction $=7.79 ; \mathrm{l}$ df; $\left.\mathrm{p}=0.0055\right)$. When grouped by BED, analysis showed that the pooled OR was $0.40(95 \%$ CI $0.13-1.22, \mathrm{z}=-1.61, \mathrm{p}=0.11)$ in patients who received a BED greater than 35 Gy and 0.64 (95\% CI $0.33-$ 1.24, $\mathrm{z}=-1.32, \mathrm{p}=0.19)$ in those who received a BED of $35 \mathrm{~Gy}$ or less without a significant interaction $\left(\chi^{2}\right.$ for interaction $=0.03 ; \mathrm{l} \mathrm{df} ; \mathrm{p}=0.86$ ).

\section{Downstaging effect}

A total of four RCTs ${ }^{12131516}$ involving 573 patients were available for evaluating the downstaging effect of CRT (fig 2). Patients who received preoperative CRT were less likely to have an advanced stage of cancer at pathological examination than were controls. Preoperative CRT was superior to surgery alone in all studies, reaching statistical significance in two RCTs. ${ }^{15}{ }^{16}$ The pooled OR was 0.43 (CI 0.26-0.72, $\mathrm{z}=-3.26, \quad \mathrm{p}=0.001) \quad(\mathrm{NNT}=5)$. Similar results were obtained when a fixed effect model was used (OR 0.44 (95\% CI 0.31-0.62); $\mathrm{z}=-4.69, \mathrm{p}=0.00001)$. In all the robust analyses the pooled estimate of the treatment effect was significant.

\section{Compliance, postoperative complications, and mortality}

Compliance with treatment was generally satisfactory. Forty five $(11.7 \%)$ of 385 patients undergoing adjuvant treatment did not complete the planned protocol, and only $25(6.5 \%)$ required a reduction in chemotherapeutic (21 patients) or irradiation dose (four patients).

The overall rate of postoperative adverse events was 39.4\% (137/348) in the CRT group and $34.3 \%(123 / 358)$ in the surgery alone group $\left(\chi^{2}=1.90 ; \mathrm{df}=1 ; \mathrm{p}=0.16\right)$. The three most frequent adverse events were respiratory complications (19.9\%), heart failure $(6.9 \%)$, and anastomotic leak $(6.9 \%)$.

The risk of postoperative mortality (within 90 days) was higher in the CRT group in five RCTs, ${ }^{12} 131516$ reaching statistical significance in one of these five trials ${ }^{16}$ in which a high dose per fraction radiation (3.7 Gy) was given (fig 3 ).

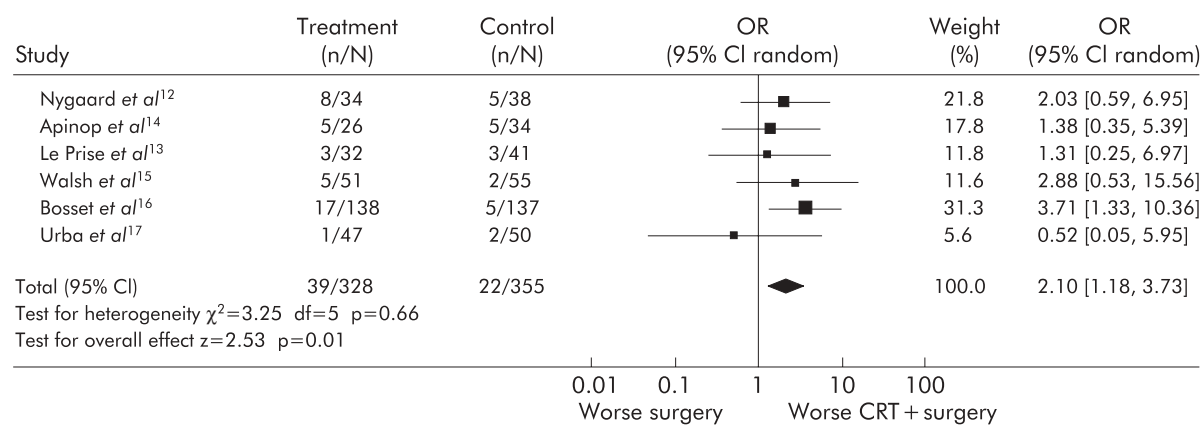

Figure 3 Postoperative mortality. Meta-analysis of six randomised controlled trials of preoperative chemoradiotherapy (CRT) for resectable oesophageal carcinoma using a random effects model. The odds ratio (OR) and $95 \%$ confidence interval (Cl) for the effect of treatment on postoperative mortality (90 day inhospital mortality) are shown on a logarithmic scale. Studies are arranged by publication year. 
Combining the data from the six RCTs, which included 683 patients, showed a significant effect of CRT on postoperative mortality (OR 2.10 (95\% CI 1.18-3.73); $\mathrm{z}=2.53, \mathrm{p}=0.01$ ) $(\mathrm{NNH}=25)$. Similar results were obtained when a fixed effect model was used (OR, 2.15 (95\% CI 1.23-3.74); z = 2.69; $\mathrm{p}=0.007)$.

Robust analysis showed a loss of significance for postoperative mortality (OR 1.62 (95\% CI $0.81-3.24$ ); $\mathrm{z}=1.36$, $\mathrm{p}=0.17$ ) once the largest trial by Bosset and colleagues ${ }^{16}$ was excluded. Moreover, sensitivity analysis performed by excluding the two RCTs ${ }^{14}{ }^{15}$ with a fraction per day dose $>2$ Gy showed a similar effect size as the robust analysis without statistical significance (OR 1.44 (95\% CI 0.67-3.08) z $=0.94$, $\mathrm{p}=0.35$ ).

\section{DISCUSSION}

This study has investigated the key clinical question of whether preoperative CRT is efficacious in treating oesophageal cancer. To our knowledge, no consensus on the type of patients amenable to chemoradiotherapy has been reached to date.

This meta-analysis of data from six RCTs showed that in resectable oesophageal cancer, preoperative CRT significantly improves three year survival versus surgery alone $(\mathrm{NNT}=10)$. Moreover, an impressive reduction in the rate of advanced oesophageal cancer (stages IIb and III) was observed in almost all trials at the time of surgery (downstaging) $(\mathrm{NNT}=5)$. Although there is evidence that chemoradiotherapy significantly increases postoperative mortality $(\mathrm{NNH}=25)$, fewer patients need to be treated to benefit from the treatment long term than need to be treated to be harmed immediately post surgery.

The effect of preoperative CRT on overall survival is much more pronounced and statistically significant in patients with adenocarcinoma, that is now more prevalent than squamous cell carcinoma in the USA ${ }^{33}$ and Western Europe, ${ }^{34}$ with most tumours located in the distal oesophagus. However, the sample size of this subgroup analysis was small (data obtained from only two trials), and caution must be exercised when interpreting results from this exploratory analysis. Nevertheless, our data strongly indicate the need for designing future trials considering the clinical difference between adenocarcinoma and squamous cell carcinoma and its potential influence on patient response to therapy. Furthermore, the current staging system for oesophageal cancer is mostly applicable to patients with squamous cell carcinomas of the upper and middle thirds of the oesophagus. To better evaluate the differential therapeutic response to CRT of patients with adenocarcinoma, this staging system should be adapted to better fit patients with adenocarcinoma, who most often present with distal oesophageal and gastrooesophageal junction tumours. In particular, patients with regional and/or coeliac axis lymphadenopathy should not necessarily be considered to have unresectable disease due to metastases.

Many efforts have been made to identify the optimal chemoradiotherapy regimen that would increase the cost effectiveness of therapy. There was considerable variation in the irradiation procedures, suggesting that worldwide accepted and standardised radiation techniques are needed to obtain comparable data on the efficacy and safety profile, particularly regarding the total and daily dose of radiation and the dose of cisplatin administered. We found that higher postoperative mortality was observed in the two RCTs ${ }^{15} 16$ in which a fraction per day dose $>2$ Gy was delivered. Furthermore, the risk of postoperative mortality was higher in the RCT by Bosset and colleagues ${ }^{16}$ in which a high dose of both radiotherapy and cisplatin was administered. On the other hand, the hyperfractionated irradiation schedule combined with a low dose of cisplatin administered in the trial by Urba and colleagues ${ }^{17}$ was more safe than the others, suggesting that a reduction of late effect achieves the best cost/effectiveness ratio. Finally, the trial by Urba and colleagues ${ }^{17}$ was the only one in which a transhiatal oesophagectomy was employed.

We believe the available information is inadequate to determine whether a concomitant regimen of chemoradiotherapy is better than induction chemotherapy followed by radiotherapy. Among the trials analysed, only that of Nygaard and colleagues ${ }^{12}$ delivered radiotherapy three weeks after completion of chemotherapy. The trials of Le Prise and colleagues $^{13}$ and Bosset and colleagues ${ }^{16}$ delivered radiotherapy within one week after chemotherapy (intermittent chemotherapy) while CRT was administered simultaneously in the remaining three RCTs. Two of these three RCTs (by Walsh and Urba) showed the highest therapeutic benefit. However, it has been postulated that induction chemotherapy may be more effective than the concomitant approach on the premise that areas of radiochemotherapy may harbour treatment resistant tumour cells and that combined toxic effects may limit the dose of drugs that can be given. Firm conclusions on the results of direct comparisons between chemoradiotherapy delivered sequentially or concomitantly are hampered by the fact that to date no trial has been performed to evaluate this variable.

Recently, a meta-analysis of individual patient data ${ }^{7}$ failed to show a statistically significant benefit of preoperative radiotherapy alone on survival. Moreover, the most recent large scale RCTs of preoperative chemotherapy as a single adjuvant treatment showed conflicting results. Our metaanalysis clearly shows that CRT as neoadjuvant treatment improves survival. We speculate that chemotherapy enhances the local effects of radiotherapy and thus decreases the likelihood of spread from the primary tumour prior to exposure of the patient to the tumour growth promoting stimulus of surgery.

The results of this retrospective analysis are subject to several limitations. Differences in the baseline severity of illness in the population of the RCTs, in the irradiation techniques, and in the chemoradiotherapy regimens may limit the accuracy of this meta-analysis. These summary results describe only between study, not between patient, variation because they reflect group averages rather than individual data. Lack of data on other potential confounders such as size and location of the tumour could also affect the accuracy of the results. The meta-analysis was performed using summary data, and more detailed treatment comparisons could be achieved with a meta-analysis of individual patient data.

Screening of the non-English literature, the extensive manual and computer searches for studies, in addition to the personal contacts made directly with principal investigators, make us confident that no important published trials were overlooked. Publication bias was probably not substantial and considered unlikely to change the direction of our pooled estimate of treatment effect. We should be particularly concerned about publication bias in settings in which small studies are being conducted.

Finally, the standard approach to inference for random effects model used in this meta-analysis including few RCTs can inflate the type I error rate. ${ }^{35}$

The available evidence is sufficient to conclude the following: (1) preoperative CRT reduces the three year overall mortality compared with surgery alone. The magnitude of the overall effect is clinically relevant. Further large scale multicentre RCTs may prove useful to substantiate the benefit on overall survival; (2) the magnitude of the downstaging effect with CRT was large; and (3) postoperative mortality 
was significantly increased by CRT. Studies addressing strategies that could potentially reduce the toxicity profile would have major implications for patients affected by oesophageal cancer.

Further RCTs in patients with oesophageal cancer investigating the efficacy and safety of induction chemotherapy in addition to preoperative chemoradiotherapy are underway by the RTOG Gastrointestinal Cancer Committee.

\section{Authors' affiliations}

F Fiorica, Cattedra di Radioterapia Oncologica, University of Modena e Reggio Emilia, Italy, and Servizio Radioterapia Oncologica, Azienda Ospedaliera Sant'Anna University of Ferrara, Italy

D Di Bona, C Cammà, Cattedra e Unità Operativa di Gastroenterologia University of Palermo, Italy, and IBIM, Consiglio Nazionale delle Ricerche, Palermo, Italy

F Schepis, Dipartimento di Medicina Interna, University of Modena and Reggio Emilia, Italy

A Venturi, A M Falchi, Cattedra di Radioterapia Oncologica, University of Modena e Reggio Emilia, Italy

A Licata, A Craxì, Cattedra e Unità Operativa di Gastroenterologia, University of Palermo, Italy

L Shahied, Fox Chase Cancer Center, Philadelphia, PA, USA

\section{REFERENCES}

1 The World Health Report 1997. Geneva: World Health Organization, 1997.

2 Faivre J, Forman D, Esteve J, et al. Survival of patients with oesophageal and gastric cancers in Europe. Eur J Cancer 1998;34:2167-75.

3 Kelsen DP, Bains M, Burt M. Neoadjuvant chemotherapy and surgery of cancer of the esophagus. Semin Surg Oncol 1990;6:268-73.

4 Muller JM, Erasmi H, Stelzner M, et al. Surgical therapy of oesophageal carcinoma. Br J Surg 1990;77:845-57.

5 Daily JM, Karnell LH, Menck HR. National Cancer Database report on oesophageal carcinoma. Cancer 1996;78:1820-8

6 Hulscher JB, van Sandick JW, de Boer AG, et al. Extended transthoracic resection compared with limited transhiatal resection for adenocarcinoma of the esophagus. N Engl J Med 2002;347:1662-9.

7 Arnott SJ, Duncan W, Gignoux M, et al. Preoperative radiotherapy for oesophageal carcinoma (Cochrane Review). London: The Cochrane Library, 2003 (issue 4)).

8 Kelsen DP, Ginsberg R, Pajak TF, et al. Chemotherapy followed by surgery compared with surgery alone for localized oesophageal cancer. N Engl J Med 1998;339:1979-84

9 Law S, Fok M, Chow S, et al. Preoperative chemotherapy versus surgical therapy alone for squamous cell carcinoma of the esophagus: a prospective randomized trial. J Thorac Cardiovasc Surg 1997;114:210-17.

10 Kok TC, van Lanschot J, Siersema PD, for the Rotterdam Oesophageal Tumor Study Group, et al. Neoadjuvant chemotherapy in operable oesophageal squamous cell cancer: final report of a phase III multicenter randomized controlled trial. Proc Am Soc Clin Oncol 1997;16:A984.

11 Medical Research Council Ooesophageal Cancer Working Party. Surgical resection with or without preoperative chemotherapy in oesophageal cancer: a randomised controlled trial. Lancet 2002;359:1727-33.

12 Nygaard K, Hagen S, Hansen HS, et al. Pre-operative radiotherapy prolong survival in operable oesophageal carcinoma: a randomized, multicenter study of pre-operative radiotherapy and chemotherapy. The second Scandinavian trial in oesophageal cancer. World J Surg 1992;16:1104-10.
13 Le Prise E, Etienne PL, Meunier B, et al. A randomized study of chemotherapy, radiation therapy, and surgery versus surgery for localized squamous cell carcinoma of the esophagus. Cancer 1994;73:1779-84.

14 Apinop C, Puttisak P, Preecha N. A prospective study of combined therapy in oesophageal cancer. Hepatogastroenterology 1994:41:391-3.

15 Walsh TN, Noonan N, Hollywood D, et al. A comparison of multimodal therapy and surgery for oesophageal adenocarcinoma. N Engl J Med 1996;335:462-7

16 Bosset JF, Gignoux M, Triboulet JP, et al. Chemoradiotherapy followed by surgery compared with surgery alone in squamous-cell cancer of the esophagus. N Engl J Med 1997;337:161-7.

17 Urba SG, Orringer MB, Turrisi A, et al. Randomized trial of preoperative chemoradiation versus surgery alone in patients with locoregional oesophageal carcinoma. J Clin Oncol 2001;19:305-13.

18 Walsh TN, Noonan N, Hollywood D, et al. Multimodality therapy versus surgery for oesophageal adenocarcinoma. A prospective randomised trial. Gastroenterology 1996;110(suppl 4):A611.

19 Ackland S, Burmeisteister BH, Smithers BM, et al. Preoperative chemoradiation (CT/RT) versus surgery alone for resectable carcinoma of esophagus: an Australasian randomised study. Proc Am Soc Clin Oncol 1998; 17:A1095.

20 Burmeister BH, Smithers BM, Fitzgerald L, et al. A randomized phase III trial of preoperative chemoradiation followed by surgery (CR-S) versus surgery alone (S) for localized resectable cancer of the esophagus. Proc Am Soc Clin Oncol 2002;20:A518.

21 Walsh TN, Noonan N, Hollywood D, et al. Multimodality therapy versus surgery for oesophageal adenocarcinoma. A prospective randomised trial. Gastroenterology 1995;108(suppl 4):A550.

22 Urba S, Orringer $M$, Turrisi A, et al. A randomized trial comparing surgery (S) to preoperative concomitant chemoradiation plus surgery in patients (pts) with resectable oesophageal cancer (CA): updated analysis. Pro Am Soc Clin Oncol, 1997; 16:A983.

23 Moher D, Cook DJ, Eastwood S, et al. Improving the quality of reports of meta-analyses of randomised controlled trials: the QUOROM statement. Quality of Reporting of Meta-analyses. Lancet 1999;27:1896-900.

24 Nicolucci A, Grilli R, Alexanian AA, et al. Quality evolution and clinical implications of randomized, controlled trials on the treatment of lung cancer: A lost opportunity for meta-analysis. JAMA 1989;262:2101-7.

25 Jüni $P$, Witschi $A$, Bloch $R$, et al. The hazards of scoring the quality of clinical trials for meta-analysis. JAMA 1999;282:1054-60.

26 Esophagus. In: American Joint Committee on Cancer: AJCC cancer Staging Manual, 5th edn. Philadelphia: Lippincott-Raven Publishers 1997:65-69.

27 DerSimonian R, Laird N. Meta-analysis in clinical trials. Control Clin Trials 1986;7:177-88.

28 Mantel N, Haenszel W. Statistical aspects of the analysis of data from retrospective studies of disease. J Natl Cancer Inst 1959;22:719-48.

29 Jaeschke R, Oxman AD, Guyatt GH. To what extent do congestive heart failure patient in sinus rhythm benefit from digoxin therapy? A systematic overview and meta-analysis. Am J Med 1990:88:279-86.

30 Barendsen GW. Dose fractionation, dose rate and iso-effect relationships for normal tissue responses. Int J Radiat Oncol Biol Phys 1982;8:1981-7.

31 Hedges LV, Olkin I. Statistical methods for meta-analysis. New York: Academic Press, 1985.

32 Yusuf S, Peto R, Lewis J, et al. Beta blockade during and after myocardial infarction: an overview of the randomized trials. Prog Cardiovasc Dis 1985;27:335-71

33 Devesa SS, Blot WJ, Fraumeni JF ir. Changing patterns in the incidence of esophageal and gastric carcinoma in the United States. Cancer 1998;83:2049-53.

34 Wayman J, Forman D, Griffin SM. Monitoring the changing pattern of esophago-gastric cancer: data from a UK regional cancer registry. Cancer Causes Control 2001;12:943-9.

35 Follman DA, Proschan MA. Valid inference in random effects meta-analysis. Biometrics 1999;55:732-7. 